\title{
Comportamentos Afetivo-Motivacionais Durante Avaliação Assistida, por Crianças com Dificuldade de Aprendizagem: Uma Proposta de Categorização ${ }^{1}$
}

\author{
Erika da Silva Ferrão \\ Centro Universitário Vila Velha \\ Elissa Orlandi Moraes \\ Sônia Regina Fiorim Enumo \\ Universidade Federal do Espírito Santo \\ Maria Beatriz Martins Linhares \\ Faculdade de Medicina de Ribeirão Preto da Universidade de São Paulo \\ Gizele Pereira de Sousa \\ Prefeitura de Domingos Martins
}

\begin{abstract}
RESUMO
A presente pesquisa objetivou construir um sistema de categorias de comportamentos observáveis de crianças com dificuldade de aprendizagem (DA), relacionado aos fatores afetivo-motivacionais, exibidos durante uma prova cognitiva de modalidade assistida. Observou-se e registraram-se os comportamentos de 10 crianças (8-10 anos) com DA, registradas em vídeo, executando uma prova assistida. Para registro cursivo desses comportamentos infantis, escolheu-se aleatoriamente uma fita por vez de 34 vídeos da atividade sendo realizada por 34 crianças até a repetição de comportamentos infantis e a diminuição da variabilidade comportamental nas situações observadas, totalizando 10 crianças observadas. Definiu-se 20 categorias, divididas em Facilitadoras e Não facilitadoras do desempenho, como Demonstrar impulsividade, Dar feedback e Pedir orientação. O nível de concordância foi calculado entre três juízes. Os resultados mostraram que 10 categorias alcançaram índices de fidedignidade acima de $70 \%$ entre pelo menos dois juízes. Estas categorias são indicadas para avaliação dos aspectos afetivo-motivacionais em avaliação assistida, tais como Corrigir pergunta/resposta após dica/ajuda, Demonstrar querer interromper a tarefa e Reclamar/responder/ atender solicitação. Estudos futuros são sugeridos a fim de testar a aplicabilidade das categorias com baixos índices de concordância.
\end{abstract}

Palavras-chave: construção de categorias; fatores afetivo-motivacionais; prova assistida.

\section{ABSTRACT \\ Affective-Motivational Behaviors During Dynamic Assessment of Children with Learning Disabilities: A Proposal of Categorization}

This research aimed to build a set of observed behaviors categories of children with learning disability related to affective-motivational factors exhibited during dynamic assessment. Ten children with learning disabilities aged 8-10 years old were videotaped while performing a dynamic testing and observation reports of these videotapes were made. In order to register these children's behavior, one tape at a time was randomly chosen from 34 videotapes, which showed the task being performed by 34 children, until the moment when the behaviors started to repeat and the behavioral variability started to decrease, concluding 10 children observed. Twenty categories were defined, divided into Facilitating and Non-facilitating of child performance, for example: Showing impulsivity, Giving feedback, Asking for help. The level of agreement was calculated among three examiners. The results showed that 10 categories assessed by at least two examiners reached reliability of ratings higher than $70 \%$. These categories are indicated to assess affective-motivational aspects in dynamic assessment, such as Correcting question/answer after hint/help; Showing willingness in stopping the task; and Complaining about answering/meeting requests. Future studies are suggested to test the applicability of the categories with low agreement ratings.

Keywords: categories' construction; affective-motivational factors; dynamic assessment. 
Há tempos a Psicologia tem se preocupado com a compreensão do processo de aprendizagem, objetivando melhorar o desempenho de crianças, principalmente daquelas referidas como tendo problemas nessa área. Apesar dos esforços realizados para elucidar o processo de aprendizagem dessas crianças a partir dos fatores cognitivos, outros fatores, denominados de afetivos e motivacionais, têm se mostrado relevantes para a investigação por profissionais e pesquisadores da área (Bandura, 1977; Costa \& Boruchovitch, 2000; Zigler, Bennett-Gates, Hodapp \& Henrich, 2002). Estudos têm sido feitos nas últimas décadas, inclusive no país, voltados para a busca de maior compreensão não só dos processos comportamentais, mas dos mecanismos emocionais ou afetivos da aprendizagem (Cruvinel \& Boruchovitch, 2004; Loureiro, 2000; Neves, 2002; Trentacosta \& Izard, 2007; entre outros) e motivacionais (Boruchovitch, 1994, 2001, 2008; Pintrich, 2003; Weiner, 1990; por exemplo). Esses mecanismos parecem afetar o desempenho de crianças com problemas nessa área tanto quanto as limitações cognitivas, sendo ainda pouco esclarecidos. A preocupação está em entender as relações existentes entre os processos de aprendizagem, desempenho escolar, mudanças cognitivas e fatores afetivo-motivacionais ou não intelectuais, entre estes, a motivação intrínseca, a ansiedade, a curiosidade e a autoeficácia, por exemplo (Tzuriel, 1991; 2001).

Alguns pesquisadores começam a estudar os fatores afetivo-motivacionais relacionados à mediação, utilizando a abordagem sociointeracionista de Vygotsky (Skuy \& Skuy, 2005) e a abordagem de Experiência de Aprendizagem Mediada, de Feüerstein e colaboradores (Feüerstein, Rand, Hoffman \& Miller, 1980). Entre os estudos na área da mediação, Haywood e Tzuriel (2002) apontam obstáculos relacionados à avaliação da aprendizagem, tais como a impulsividade e o autoconceito empobrecido em relação à sua condição de aprendiz, e as variáveis motivacionais. Alguns desses obstáculos, se removidos, possibilitariam a identificação e o desenvolvimento de certas habilidades cognitivas. Esses autores, ao indicarem estratégias originadas da mediação relevantes para a situação de ensino, por melhorarem o desempenho de crianças com necessidades educacionais especiais, citam algumas estratégias que estariam relacionadas aos fatores afetivos envolvidos no comportamento do mediador em sua relação ao aprendiz, como a atenção especial às barreiras para o investimento em aprendizagem e expectativa de sucesso. Destaca-se, assim, o papel do mediador em identificar, na situação de mediação, os comportamentos infantis relacionados aos fatores afetivo-motivacionais observados ao longo de uma tarefa realizada pela criança.

Apesar de estar bem estabelecida a relação entre os fatores afetivo-motivacionais e as dificuldades de aprendizagem em contexto de mediação, ainda há dificuldade de identificar, a partir da observação, os comportamentos infantis relacionados aos aspectos afetivos e motivacionais em tal contexto (Ferrão \& Enumo, 2008). Da mesma forma, são necessários estudos sobre como intervir nesses aspectos para potencializar o desempenho das crianças em situações educacionais, principalmente de ensino-aprendizagem e avaliativas, em que esses parecem ser bastante importantes para se entender o desempenho apresentado pela criança.

Para o estudo dos fatores afetivo-motivacionais do desempenho de crianças com dificuldade de aprendizagem em situação de mediação, a avaliação cognitiva assistida tem se mostrado muito eficaz, por possuir características que tornam possível a observação e investigação desses fatores não intelectuais do examinando, verificados em uma situação de aprendizagem mediada. Nascida da insatisfação com a abordagem psicométrica tradicional de avaliação, a Avaliação Assistida ou Dinâmica (Dynamic Assessment) surgiu na década de 70 do século XX como uma nova forma de avaliação psicológica, proposta por Feüerstein e cols. (Haywood, 2008; Haywood \& Lidz, 2007; Linhares \& Enumo, 2007; Linhares, Escolano \& Enumo, 2006; Sternberg \& Grigorenko, 2002). Está fundamentada em conceitos, como o de potencial de aprendizagem e aprendizagem mediada, e na teoria da Experiência de Aprendizagem Mediada de Feüerstein e cols. (Feüerstein \& cols., 1980; Feüerstein, Rand \& Hoffman, 1979). Entre as características da avaliação assistida, podem ser citadas: (a) inclui uma situação de miniaprendizagem durante a avaliação, denominada "fase de assistência", em que o mediador pode fornecer à criança diferentes tipos de ajuda, desde sugestões a instruções passo a passo, a fim de identificar, além do desempenho real/atual, o potencial para a aprendizagem da criança em situação de interação com uma pessoa mais capaz, no caso, o professor, por exemplo; e (b) permite um contexto de aplicação em que outras pessoas, como pais e professores, podem observar a execução da prova. Essa avaliação mais ampla e esse contexto de aplicação não são propostos na avaliação psicométrica tradicional, residindo aqui uma de suas críticas (Haywood, 2008; Haywood \& Lidz, 2007). 
$\mathrm{Na}$ investigação dos fatores afetivo-motivacionais exibidos em situação de mediação, há algumas tentativas de operacionalização, como as propostas por Lidz (2000, 2003), Jensen (1997), Tzuriel (1991, 2001) e Tzuriel, Samuels e Feuerstein (1988). O estudo de Jensen (1997), por exemplo, propõe vinte categorias de fatores não intelectuais, relacionadas aos atributos de personalidade e aos motivos e necessidades, verificados a partir da análise da mediação, tais como: necessidade por domínio, locus de controle, necessidade por novidade, desejo por aprovação, ficar na defensiva, habilidade para confiar em seus próprios pensamentos e sentimentos.

Os fatores não intelectuais, que podem influenciar negativamente o desempenho da criança, específicos ao procedimento de avaliação assistida, ou seja, de uma determinada situação de experiência de aprendizagem mediada, também foram identificados por Lidz (2000, 2003), Tzuriel (1991; 2001) e Tzuriel, Samuels e Feuerstein (1988). Tzuriel (1991), embasado em trabalhos anteriores sobre os conceitos de afetividade e motivação, de locus de controle e de autodeterminação, por exemplo, propõe e define sete fatores não intelectuais específicos à área de aprendizagem mediada:

1) Acessibilidade à mediação: problemas nesta área podem ser observados pela manifestação de ativa rejeição às tentativas do mediador para ensinar, ou pelo afastamento passivo da situação pela criança;

2) Necessidade de domínio: consiste na persistência da criança na tarefa, tentativa para trabalhar independentemente, sem ajuda do examinador;

3) Tolerância à frustração: expressada pela fácil desistência após dificuldade na experiência, ou por mostrar sinais de irritação quando solicitado a resolver uma outra tarefa ou a explicar e elaborar uma resposta;

4) Locus de controle interno ou externo: percepção individual de ser responsável pelos resultados de seu comportamento e pelo controle sobre os eventos da vida, ou atribuição de causalidade em fontes externas;

5) Medo de fracasso e atitude defensiva: geralmente presentes em atividade que relembram à criança fracassos anteriores ou que se mostram complicadas para enfrentar;

6) Confiança na resposta correta: refere-se à crença da criança de que a resposta dada é correta, mesmo quando desafiada ou solicitada a explicar;

7) Vitalidade e sentido de alerta: compreende o nível de atividade, energia, vivacidade, atenção e interesse que a criança mostra na interação com o mediador.
Tzuriel e Samuels (2000) procuraram testar a validade dessas categorias a serem aplicadas ao longo de várias provas assistidas. Aplicaram oito testes da bateria Learning Potential Assessment Device (LPAD), proposto por Feüerstein em 1979, em 35 crianças com idade entre 12 e 14 anos, com e sem dificuldade de aprendizagem, associada ou não com déficit de atenção e hiperatividade, e deficientes mentais educáveis. Obtiveram índices de concordância de $80 \%$ em 11 categorias afetivo-motivacionais, que incluem as sete categorias já citadas, sendo a categoria Medo de falhar e atitude defensiva separada em duas categorias (Defensiva; Medo de falhar). Além disso, foram acrescentadas mais três categorias, a saber: 1) Necessidade de explorar a tarefa - curiosidade; 2) Prazer na atividade; e 3) Necessidade de continuar a trabalhar em outras tarefas gerais. Essas categorias foram observadas ao longo das provas assistidas e avaliadas por três juízes. De acordo com os autores, cinco das 11 categorias (Tolerância à frustração, Locus de controle interno, Defensiva, Acessibilidade à mediação, e Vitalidade e alerta) obtiveram baixo índice de concordância quando não se considerou a pontuação zero como possibilidade, sendo o menor índice obtido pela categoria Defensiva (25\%). Os maiores índices alcançados foram nas categorias Medo de fracasso, com 91,3\% de concordância, e Trabalhar de forma independente $(86,2 \%)$.

Outra avaliação dessas mesmas categorias foi feita por Paula e cols. (Paula, 2004; Paula \& Enumo, 2007) em crianças brasileiras com problemas de comunicação, em idade escolar. Os índices obtidos em algumas categorias foram baixos (Tolerância à frustração e Atitude defensiva, com $40 \%$, por exemplo), enquanto outras categorias apresentaram altos índices de concordância entre os juízes, como Acessibilidade à mediação (80\%) e Necessidade de explorar a tarefa (Paula, 2004). Contudo, essas categorias se mostraram amplas e demasiadamente teóricas para uso em pesquisa, tanto que, concordando com Tzuriel e Samuels (2000), Paula e Enumo (2007) sugerem a realização de novas pesquisas na área da aprendizagem mediada, para tornar essas categorias mais objetivas e claras.

Nesse sentido, baseado nas definições dos fatores afetivo-motivacionais ou não intelectuais propostos por autores da área (Jensen, 1997; Lidz, 2000, 2003; Tzuriel, 1991; Tzuriel \& cols., 1988), o presente estudo teve por objetivo elaborar um conjunto de categorias de comportamento de crianças em idade escolar, relacionados a fatores afetivo-motivacionais, presentes no contexto de aprendizagem mediada, específico 
às peculiaridades de uma prova de habilidades cognitivas, no contexto de um procedimento de avaliação assistida pelo examinador.

\section{MÉTODO}

\section{Participantes e fontes documentais}

Os dados analisados foram obtidos em registros em vídeo de 10 sessões (tempo médio/sessão $=41$ minutos), totalizando 410 minutos de gravação da aplicação do Jogo de Perguntas de Busca com Figuras Diversas (Linhares \& Gera, 2006), em 10 crianças, com idade entre 8 a 10 anos (idade média de 8,8 anos), sendo seis delas meninas. Estas foram selecionadas de uma amostra de 34 alunos (8-12 anos, idade média: 9 anos; 20 meninas), com indicação de dificuldade de aprendizagem (DA), por apresentarem baixo desempenho no Teste de Desempenho Escolar (Stein, 1994); frequentavam a $2^{\mathrm{a}}$ série (11 meninos e 8 meninas) e $3^{\mathrm{a}}$ série ( 12 meninas e 3 meninos) de uma escola pública de uma capital da Região Sudeste, onde os dados foram coletados (março-julho/2002), por um pesquisador, com auxílio para filmagem. O grupo apresentou um QI médio no WISC de 92 (AV=77-105); nas Matrizes Progressivas Coloridas de Raven - Escala Especial (Angelini, Alves, Custódio, Duarte \& Duarte, 1999), a maioria dos alunos $(55.8 \%)$ obteve classificação "média" e "abaixo da média" (AV= abaixo da média a superior); no PBFD (Linhares \& Gera, 2006) e a mediana do perfil cognitivo foi "alto-escore" $(\mathrm{AV}=$ baixo-escore a alto-escore), conforme classificação proposta por Linhares, Santa Maria e Escolano (2006).

Esses registros em vídeo contêm a aplicação da seguinte prova de habilidades cognitivas, com procedimento assistido, descrita a seguir.

\section{Material e instrumentos}

O contexto analisado nas gravações incluía a realização do Jogo de Perguntas de Busca com Figuras Diversas - PBFD (Linhares \& Gera, 2006) - uma prova assistida, que avalia as estratégias adotadas pela criança para elaborar questões de busca de informações com restrição de alternativas e raciocínio de exclusão em situação de resolução de problemas. É organizada em 21 arranjos, com oito figuras coloridas cada um, sendo aplicada em cinco fases: 1) exemplo (EXE) - um arranjo; 2) sem ajuda (SAJ) - quatro arranjos; 3) assistência (ASS)- oito arranjos; 4) manutenção (MAN) - quatro arranjos; e 5) transferência (TRF) - quatro arranjos. A criança deve descobrir qual a figura em que o examinador está pensando, por meio de perguntas de busca, com exclusão de alternativas. A criança pode fazer os seguintes tipos de pergunta: (a) relevante - explora um dos atributos da figura possibilitando o levantamento de informações novas sobre aquela a ser descoberta; (b) irrelevante - feita sobre algum atributo já descoberto através das perguntas anteriores, sendo, portanto, desnecessária, na medida em que não traz informação nova sobre a figura escolhida pelo examinador; (c) incorreta - outras perguntas que não exploram os atributos das figuras; e (d) repetida. Para que a criança consiga descobrir a figura correta, ela deve fazer, em cada arranjo, no mínimo, três a quatro perguntas relevantes, dependendo do arranjo em questão. As respostas da criança, por sua vez, são analisadas como tipos de tentativa de solução: (a) correta; (b) incorreta; e (c) correta-ao-acaso - quando a resposta é encontrada sem terem sido feitas as perguntas necessárias para a descoberta da mesma.

Essa prova assistida, no formato "estruturado", usado em situação de pesquisa, o examinador/mediador pode oferecer os seguintes níveis de ajuda, na fase de ASS (Linhares \& Gera, 2006) - nível 1: feedback; nível 2: análise comparativa; nível 3: exemplo de pergunta relevante; nível 4: separação dos cartões/figura já excluídos; nível 5: demonstração de um modelo de uso da estratégia.

Para a análise dos dados, foi utilizado um protocolo de registro cursivo das sessões, contendo informações, tais como: a identificação, com nome, idade e sexo, descrição do ambiente físico e social, duração da sessão e o registro propriamente dito. Foram utilizados os seguintes equipamentos: cronômetro, televisão colorida, videocassete e computador.

\section{Procedimento}

Na primeira fase, foi feita a seleção das sessões para análise, considerando que cada fita continha a gravação de uma só criança realizando a prova assistida. Foram feitas observações de registros em vídeo dos comportamentos verbais e não verbais das crianças em interação com o mediador e vice-versa, e registro cursivo ao longo da resolução das cinco fases da prova assistida (exemplo, sem ajuda, assistência, manutenção e transferência).

De um total de 34 crianças com DA, avaliadas em 34 sessões de aplicação do PBFD, foram selecionadas aleatoriamente 10 fitas para registro. Cada observação era caracterizada pelo registro contínuo de aspectos do 
comportamento, tais como postura corporal da criança ao longo da realização da tarefa e verbalizações da criança e do mediador desde o início até o encerramento da atividade, relacionadas ou não à tarefa. A esses dados do protocolo, foram acrescentados: o tempo decorrido, em minutos, ao longo de toda a sessão; as fases da prova e a identificação de cada prancha em cada fase; além do desempenho cognitivo apresentado pela criança em cada prancha.

As categorias foram construídas paralelamente à realização contínua dos 10 registros cursivos. Antes de ser finalizado, o conjunto de categorias sofreu alterações ao ser aplicado nos novos registros e no processo de adaptação das descrições dos comportamentos infantis observados, tomando por base os fatores afetivos e motivacionais do desempenho infantil propostos por autores da área.

$\mathrm{Na}$ fase seguinte, a cada grupo de três a quatro registros que iam sendo finalizados, eram identificadas e listadas exaustivamente as unidades de análise (Batista, 1999), que, posteriormente, foram agrupadas, seguindo o critério de semelhança funcional entre si ou de certas características relacionadas aos fatores afetivo-motivacionais ao qual eles estavam ligados. Originaram-se, assim, as primeiras categorias. Para a nomeação e a descrição dessas categorias, foram utilizadas definições de categorias e verbos propostos por pesquisadores da área da interação (Batista, 1999; Santana, Otta \& Bastos, 1993; Sigolo \& Alves, 1998; Silva \& Salomão, 2002; por exemplo). Contudo, essas definições foram adaptadas às características da prova assistida em questão (PBFD). Por exemplo, foram incluídas: a descrição dos modos de resolução das diferentes tarefas assistidas e a situação em que o examinador oferece alguma instrução inicial, assistência ou verbaliza comandos, acréscimos estes necessários para o caso de algumas tarefas assistidas. As descrições das categorias afetivo-motivacionais elaboradas continham descrições que mostravam serem mutuamente exclusivas.

Quanto à dimensão temporal das categorias, percebia-se que algumas delas eram instantâneas, ou seja, para que ela fosse identificada e registrada, era neces- sário identificar ocorrência ou não ocorrência de unidades de análise que a representavam, a exemplo da categoria Pedir orientação. Já outras categorias, como Demonstrar inquietude, para serem identificadas, precisavam ser observadas ao longo de mais de um evento.

Assim que outros grupos de registro ficavam prontos e o mesmo procedimento se repetia, certas unidades de análise foram sendo incluídas nas categorias já existentes, não sendo necessária a construção de uma nova categoria. Assim que se verificou a repetição de comportamentos e a diminuição da variabilidade comportamental nas situações observadas, interrompeu-se o registro cursivo (Danna \& Matos, 1982; Fagundes, 1982), fato que aconteceu durante a observação da décima fita. Com este procedimento, foi possível observar em vídeo e registrar os comportamentos de 10 crianças e do examinador em 10 sessões de aplicação do PBFD e também definir um conjunto de 20 categorias descritivas do comportamento da criança em interação. Essas categorias foram divididas em Facilitadoras e Não facilitadoras do desempenho, para diferenciar os comportamentos que permitiam ou não um bom desempenho da criança ao longo das fases do jogo, baseado em Santa Maria e Linhares (1999).

Finalmente, foi realizado o cálculo da concordância (índice de $70 \%$ ) com $10 \%$ da amostra das 34 crianças (3 crianças) entre três juízes treinados (Fagundes, 1982). Foi considerado o zero como concordância, visto que a situação avaliada permitia o aparecimento de todas as categorias (Tzuriel \& Samuels, 2000).

\section{RESULTADOS}

Foram identificadas e definidas 20 categorias descritivas relacionadas aos fatores afetivo-motivacionais, sendo oito compostas por comportamentos facilitadores e 12 não facilitadores do desempenho na prova assistida. As categorias de comportamentos facilitadores e suas definições encontram-se na Tabela 1 . 
TABELA 1

Categorias de Comportamentos Facilitadores do Desempenho Infantil e suas Definições

\begin{tabular}{|c|c|}
\hline Categorias de comportamentos & Definição \\
\hline \multicolumn{2}{|l|}{ Facilitadores } \\
\hline 1. Atender ordem & $\begin{array}{l}\text { C acata ordem do M para voltar à tarefa após exibir comportamento perturbador ou distrair-se } \\
\text { com o ambiente. }\end{array}$ \\
\hline $\begin{array}{l}\text { 2. Corrigir pergunta ou resposta } \\
\text { após dica/ajuda }\end{array}$ & $\begin{array}{l}\text { Após dica, como perguntar se a criança tem certeza ou pedir para que olhe as figuras nova- } \\
\text { mente ou atentamente, ou algum nível de ajuda de } \mathrm{M}, \mathrm{C} \text { corrige a pergunta/resposta feita/dada } \\
\text { anteriormente. }\end{array}$ \\
\hline $\begin{array}{l}\text { 3. Corrigir pergunta/resposta } \\
\text { espontaneamente }\end{array}$ & Espontaneamente, $\mathrm{C}$ corrige a pergunta/resposta feita/dada anteriormente. \\
\hline 4. Dar feedback & $\begin{array}{l}\text { Imediatamente após pergunta, comentário ou solicitação de M relacionados à tarefa ou outro } \\
\text { assunto escolhido por M, C responde ou concorda, emitindo comportamentos verbais e/ou não } \\
\text { verbais. }\end{array}$ \\
\hline 5. Demonstrar afeto & $\begin{array}{l}\text { C demonstra afetividade, com comportamentos como sorrir para } \mathrm{M} \text {, respondendo ou não à } \\
\text { demonstração de afeto de } \mathrm{M} \text {. }\end{array}$ \\
\hline $\begin{array}{l}\text { 6. Resolver a tarefa seguindo } \\
\text { instruções }\end{array}$ & $\begin{array}{l}\text { Após mando, instrução ou pergunta de } \mathrm{M} \text { relacionados à tarefa, C executa a tarefa segundo as } \\
\text { instruções. }\end{array}$ \\
\hline $\begin{array}{l}\text { 7. Exibir confiança na resposta } \\
\text { correta }\end{array}$ & $\begin{array}{l}\text { Após questionamento de } \mathrm{M}, \mathrm{C} \text { confirma verbalmente ou não a pergunta/resposta por ela fei- } \\
\text { ta/dada anteriormente. }\end{array}$ \\
\hline 8. Pedir orientação & $\begin{array}{l}\text { C solicita informações sobre a tarefa, esclarecimento de dúvidas sobre alguma característica } \\
\text { de atributo em específico ou sobre o modo de como realizar a tarefa. }\end{array}$ \\
\hline
\end{tabular}

Nota. $\mathrm{C}=$ criança; $\mathrm{M}=$ mediador.

Na Tabela 2, é possível ver um exemplo de cada categoria de comportamento facilitador do desempenho infantil.

TABELA 2

Categorias de Comportamentos Facilitadores do Desempenho Infantil e Exemplos

\begin{tabular}{|c|c|}
\hline Categorias de comportamentos & Exemplo \\
\hline \multicolumn{2}{|l|}{ Facilitadores } \\
\hline 1. Atender ordem & $\begin{array}{l}\text { Após } M \text { chamar atenção de } C \text {, enquanto } C \text { olhava em direção à porta, } C \text { olha em direção às } \\
\text { figuras. }\end{array}$ \\
\hline $\begin{array}{l}\text { 2. Corrigir pergunta ou resposta } \\
\text { após dica/ajuda }\end{array}$ & $\begin{array}{l}\text { Após } M \text { repetir a pergunta incorreta feita por } C \text {, durante a fase de assistência, } C \text { corrige a } \\
\text { pergunta. }\end{array}$ \\
\hline $\begin{array}{l}\text { 3. Corrigir pergunta/resposta } \\
\text { espontaneamente }\end{array}$ & C corrige pergunta incorreta feita anteriormente sobre um atributo. \\
\hline 4. Dar feedback & Após M perguntar se $\mathrm{C}$ está afirmando ou perguntando, $\mathrm{C}$ responde que está afirmando. \\
\hline 5. Demonstrar afeto & Após M sorrir, olhando em direção a C, C sorri e balança a cabeça afirmativamente. \\
\hline $\begin{array}{l}\text { 6. Resolver a tarefa seguindo } \\
\text { instruções }\end{array}$ & $\begin{array}{l}\text { Enquanto } \mathrm{M} \text { apresenta o terceiro arranjo da fase de manutenção, } \mathrm{C} \text { verbaliza comentário sobre } \\
\text { os atributos da figura. }\end{array}$ \\
\hline $\begin{array}{l}\text { 7. Exibir confiança na resposta } \\
\text { correta }\end{array}$ & Após $\mathrm{C}$ fazer pergunta correta, $\mathrm{M}$ pergunta confirmação da pergunta feita por $\mathrm{C}$ que a confirma. \\
\hline 8. Pedir orientação & $\begin{array}{l}\text { Após } M \text { responder pergunta feita por } C, C \text { pede esclarecimento preciso sobre cor impressa na } \\
\text { figura. }\end{array}$ \\
\hline
\end{tabular}

Nota. $\mathrm{C}=$ criança; $\mathrm{M}=$ mediador . 
Percebe-se que as descrições apresentadas em alguns dos exemplos apresentam peculiaridades da prova assistida (PBFD), tal como o exemplo da categoria Resolver a tarefa seguindo instruções, especificando qual arranjo resolvido pela criança e que são os atributos da figura da prova que devem ser analisados pela criança.

As definições das categorias de comportamentos não facilitadores encontram-se na Tabela 3, apresentada a seguir.

TABELA 3

Categorias de Comportamentos Não Facilitadores do Desempenho Infantil e suas Definições

\begin{tabular}{ll}
\hline Categorias de comportamentos & \\
\hline Não facilitadores & Definição \\
\hline 9. Demonstrar cansaço & $\begin{array}{l}\text { C exibe comportamentos verbais ou não verbais que indicam cansaço, como bocejar, verbalizar ou } \\
\text { responder que está com sono ou cansado. }\end{array}$ \\
\hline $\begin{array}{l}\text { 10. Demonstrar querer } \\
\text { interromper a tarefa }\end{array}$ & $\begin{array}{l}\text { Após questionamento ou não de M, C exibe comportamentos verbais ou não verbais que indicam } \\
\text { querer informações sobre o término da tarefa ou expressam querer interromper, como perguntar } \\
\text { quando termina, se pode ir embora, afirma ou responde que não quer continuar. }\end{array}$ \\
\hline 11. Demonstrar inquietude & $\begin{array}{l}\text { C emite comportamentos não verbais que indicam desassossego ou inquietude, como me- } \\
\text { xer/balançar as mãos, roer unhas, morder gola da camisa, mexer/balançar as pernas/mexer-se na }\end{array}$ \\
cadeira.
\end{tabular}

12. Demonstrar impulsividade $\quad \mathrm{C}$ apresenta comportamentos que demonstram falta de atenção, concentração e tempo adequado de análise da melhor pergunta/resposta a ser feita/dada, escolhendo figura correta-ao-acaso ou incorreta.

13. Demonstrar timidez $\quad$ C exibe comportamentos verbais e não verbais que indicam retraimento ou timidez, como encolher os ombros, emitir voz baixa e/ou ininteligível, e juntar e/ou manter mãos entre as pernas.

14. Distrair-se com $0 \quad$ Durante ou após instrução ou outras verbalizações de $M$ sobre a tarefa, $C$ atenta para 0 ambiente ambiente físico e social, emitindo comportamentos como olhar, comentar sobre atributos do ambiente ou outras pessoas presentes.

15. Reprovar-se diante de erro Após confirmação de $M$ de que $C$ não emitiu o comportamento esperado na tarefa, $C$ exibe comportamentos de autorreprovação.

16. Exibir comportamento $\quad$ C exibe comportamento verbal ou não verbal, que interrompe ou atrapalha a instrução ou outros disruptivo comentários e solicitações feitas por $\mathrm{M}$.

17. Iniciar assuntos $\quad$ C verbaliza outros assuntos não relacionados à tarefa. incompatíveis com a tarefa

18. Descumprir instruções $\quad$ Após oferecimento de diferentes níveis de ajuda, $C$ insiste em perguntar/responder, sem cumprir as instruções iniciais apresentadas na fase de assistência por $M$.

19. Reclamar por ter que res- Diante de solicitação ou pergunta de M, C emite comportamentos verbais ou não verbais de reclaponder/atender solicitação mação, antes de atender.

20. Recusar-se a respon- $\quad$ Após pergunta ou instrução de $M, C$ verbaliza que não atenderá à solicitação. der/atender solicitação

Nota. $\mathrm{C}=$ criança; $\mathrm{M}=$ mediador .

Os exemplos das categorias de comportamentos não facilitadores encontram-se na Tabela 4. 
TABELA 4

Categorias de Comportamentos Não Facilitadores do Desempenho Infantil e Exemplos

\begin{tabular}{|c|c|}
\hline Categorias de comportamentos & Exemplos \\
\hline \multicolumn{2}{|l|}{ Não facilitadores } \\
\hline 9. Demonstrar cansaço & $\begin{array}{l}\text { Enquanto M solicita que ela preste atenção ao longo da explicação da fase de assistência, C } \\
\text { boceja. }\end{array}$ \\
\hline $\begin{array}{l}\text { 10. Demonstrar querer interromper a } \\
\text { tarefa }\end{array}$ & Enquanto M apresenta último arranjo da fase de manutenção, C pergunta "É a última?" \\
\hline 11. Demonstrar inquietude & $\begin{array}{l}\text { Após M dar a instrução inicial no arranjo de exemplo e solicitar para que } \mathrm{C} \text { explique a tarefa, } \mathrm{C} \\
\text { esfrega as mãos nas pernas. }\end{array}$ \\
\hline 12. Demonstrar impulsividade & $\begin{array}{l}\text { Após } M \text { responder pergunta relevante feita por } C \text { e sugerir que } C \text { olhasse para as figuras e } \\
\text { continuasse a fazer perguntas, } C \text { aponta resposta correta-ao-acaso. }\end{array}$ \\
\hline 13. Demonstrar timidez & C verbaliza algo ininteligível (M solicita que $\mathrm{C}$ refaça a pergunta em tom mais alto). \\
\hline 14. Distrair-se com o ambiente & Após M instruir para começar, C olha em direção à porta. \\
\hline 15. Reprovar-se diante de erro & $\begin{array}{l}\text { Após } M \text { responder que a pergunta feita por } C \text { está errada, } C \text { balança a cabeça negativamente } \\
\text { olhando em direção às figuras. }\end{array}$ \\
\hline 16. Exibir comportamento disruptivo & $\begin{array}{l}\text { C deita a cabeça sobre os braços cruzados na mesa enquanto faz uma pergunta irrelevante e } \\
M \text { chama sua atenção, pede que } C \text { levante a cabeça, sente corretamente e preste mais aten- } \\
\text { ção. }\end{array}$ \\
\hline $\begin{array}{l}\text { 17. Iniciar assuntos incompatíveis } \\
\text { com a tarefa }\end{array}$ & $\begin{array}{l}\text { Enquanto } M \text { espera alguma pergunta a ser feita pela criança, } C \text { verbaliza sobre algo para a } \\
\text { professora. }\end{array}$ \\
\hline 18. Descumprir instruções & $\begin{array}{l}\text { Após M oferecer ajuda nível } 4 \text { durante arranjo } 7 \text { da fase de assistência, C faz pergunta incorre- } \\
\text { ta: "É esse?" }\end{array}$ \\
\hline $\begin{array}{l}\text { 19. Reclamar por ter que respon- } \\
\text { der/atender solicitação }\end{array}$ & $\begin{array}{l}\text { Enquanto M apresenta primeiro arranjo da fase de assistência, C pergunta "é de novo?" Após } \\
\text { M confirmar, C verbaliza um palavrão, e faz uma pergunta incorreta. }\end{array}$ \\
\hline $\begin{array}{l}\text { 20. Recusar-se a responder/atender } \\
\text { solicitação }\end{array}$ & Após solicitação de M para fazer a pergunta, C verbaliza que não quer fazer a pergunta. \\
\hline
\end{tabular}

Analisando a Tabela 3 e a Tabela 4, percebe-se que as categorias de comportamentos não facilitadores do desempenho infantil, na prova assistida (PBFD), estão em maior número, quando comparadas às categorias facilitadoras.
Finalmente, após a definição dessas categorias, foi feito o cálculo de concordância para cada categoria, considerando concordância o índice de $70 \%$, por cada dupla de análise (AB-BC-AC) de três juízes treinados (ver Tabela 5). 
TABELA 5

Cálculo de Concordância para Cada uma das 20 Categorias, por Dupla de Análise (AB-BC-AC) de Três Juízes Treinados. $(n=20)$

\begin{tabular}{lccc}
\multicolumn{1}{c}{ Categorias de comportamentos } & $\mathrm{A}-\mathrm{B}$ & $\mathrm{B}-\mathrm{C}$ & $\mathrm{A}-\mathrm{C}$ \\
& $(\%)$ & $(\%)$ & $(\%)$ \\
\hline Facilitadores & & & \\
\hline 1. Atender ordem & 33 & 66 & $100^{*}$ \\
2. Corrigir pergunta ou resposta após dica/ajuda & $79^{*}$ & $83^{*}$ & $91^{*}$ \\
3. Corrigir pergunta/resposta espontaneamente & 55 & 66 & $88^{*}$ \\
4. Dar feedback & 50 & $70^{*}$ & $74^{*}$ \\
5. Demonstrar afeto & $81^{*}$ & $88^{*}$ & $91^{*}$ \\
6. Resolver a tarefa seguindo instruções & 57 & 58 & $89^{*}$ \\
7. Exibir confiança na resposta correta & 66 & 50 & $100^{*}$ \\
8. Pedir orientação & $67^{*}$ & $75^{*}$ & $88^{*}$ \\
\hline Não facilitadores & & & \\
\hline 9. Demonstrar cansaço & $100^{*}$ & $66^{*}$ & $100^{*}$ \\
10. Demonstrar querer interromper a tarefa & $100^{*}$ & $100^{*}$ & $100^{*}$ \\
11. Demonstrar inquietude & 41 & 62 & 49 \\
12. Demonstrar impulsividade & 01 & $88^{*}$ & 22 \\
13. Demonstrar timidez & 66 & $100^{*}$ & 66 \\
14. Distrair-se com o ambiente & 04 & 09 & $83^{*}$ \\
15. Reprovar-se diante de erro & 66 & 33 & 66 \\
16. Exibir comportamento disruptivo & $100^{*}$ & $100^{*}$ & $100^{*}$ \\
17. Iniciar assuntos incompativeis com a tarefa & $66^{*}$ & $100^{*}$ & $100^{*}$ \\
18. Descumprir instruções & $76^{*}$ & 53 & 09 \\
19. Reclamar por ter que responder/atender solicitação & $100^{*}$ & $100^{*}$ & $100^{*}$ \\
20. Recusar-se a responder/atender solicitação & $100^{*}$ & $100^{*}$ & $100^{*}$ \\
\hline
\end{tabular}

Nota. *índice igual ou maior que $70 \%$.

Tal como mostra a Tabela 5, das 20 categorias, seis tiveram avaliação maior que $70 \%$ pelos três juízes Corrigir pergunta/resposta após dicalajuda; Demonstrar afeto; Demonstrar querer interromper a tarefa; Exibir comportamento disruptivo; Reclamar por ter que responder/atender solicitação; e Recusar-se a responder/atender solicitação. Quatro categorias alcançaram mais de $70 \%$ de concordância por, pelo menos, duas duplas de juízes - Dar feedback; Pedir orientação; Demonstrar cansaço; e Iniciar assuntos incompativeis com a tarefa. As demais 10 categorias tiveram baixo índice para todas as três duplas de juízes.

\section{DISCUSSÃO}

A proposta da presente pesquisa, que tem caráter -metodológico, foi operacionalizar os fatores afetivomotivacionais propostos por autores da área, com a definição de categorias comportamentais, observáveis de crianças com dificuldade de aprendizagem realizando uma prova assistida. Os resultados da análise de concordância das categorias permitem algumas considerações em relação às possibilidades e dificuldades que estudiosos de diferentes áreas ainda encontram para avaliar os fatores afetivos e motivacionais dessas crianças a partir do método observacional (Tzuriel \& Samuels, 2000; Zigler \& cols., 2002).

$\mathrm{O}$ baixo índice de concordância entre os juízes na avaliação de algumas categorias, como Demonstrar timidez e Demonstrar impulsividade, provavelmente confirma a dificuldade de se avaliar fatores afetivo-motivacionais que exigem do avaliador maior capacidade de uma habilidade cognitiva, denominada de pensamento hipotético-inferencial (Feüerstein \& cols., 1980). Nesse sentido, as categorias com baixo índice de concordância precisam ter suas definições aperfeiçoadas, com a inclusão de mais detalhes na descrição dos comportamentos da criança. 
Por sua vez, as categorias que alcançaram bom índice de concordância entre os juízes (acima de 70\%) podem ser uma alternativa de análise de alguns comportamentos infantis relacionados aos fatores afetivos e motivacionais, exibidos durante a avaliação assistida. Para o avaliador que observa o desempenho dessas crianças, essas categorias estão mais claras e objetivas do que aquelas propostas por autores da área (Tzuriel, 2001), Um exemplo é a categoria aqui construída $D e-$ monstrar querer interromper a tarefa, que permite uma análise mais objetiva do comportamento relacionado ao fator afetivo-motivacional, proposto por Tzuriel (2001) - vitalidade e sentido de alerta; assim como a categoria Corrigir-se espontaneamente, relacionada ao conceito de locus de controle interno.

Além disso, as categorias propostas nesta pesquisa são mais adaptadas às peculiaridades de um procedimento assistido por incluir alguns tipos de perguntas feitas pelas crianças durante a prova e os diferentes níveis de ajuda oferecidos pelo mediador. Como exemplo, criou-se a categoria Corrigir pergunta/resposta após dica/ajuda, que inclui, em sua definição, a variável "nível de ajuda" oferecida pelo mediador durante o procedimento assistido em questão (PBFD). Assim, elas podem ser utilizadas pelo avaliador que aplicar tarefas envolvendo habilidades cognitivas, usando esse procedimento assistido ou outros que possuam as mesmas características do PBFD, a exemplo do Jogo de Perguntas de Busca com Figuras Geométricas (PBFG) (Linhares, 2006) e do Jogo de Perguntas de Busca com Figuras Geométricas para Deficientes Visuais (PBFG-DV) (Enumo \& Batista, 2006). Esta condição evidencia uma vantagem do uso das categorias construídas nesta pesquisa sobre outras categorias usualmente utilizadas por pesquisadores da área da interação infantil, as quais não consideram as peculiaridades de um instrumento de avaliação assistida, como ocorre com os sistemas de categorias de interação propostos por Sigolo e Alves (1998), Silva e Salomão (2002).

Até o momento, esta pesquisa permitiu a construção confiável de 10 categorias de comportamentos, facilmente observáveis, relacionados aos fatores afetivo-motivacionais do desempenho cognitivo de crianças com dificuldade de aprendizagem realizando uma prova dinâmica ou assistida. Tais categorias facilitam a análise do mediador dos fatores envolvidos com o desempenho infantil durante a avaliação assistida, quando comparadas também com as categorias propostas por autores da área (Lidz, 2000, 2003; Tzuriel, 2001).
Nesse sentido, é importante que novos estudos sejam realizados para testar a aplicabilidade das categorias que tiveram bom índice de concordância, $\mathrm{e}$, principalmente, rever as categorias com baixo índice de concordância, para que essas possam ser melhor definidas, até alcançar um índice maior de confiabilidade.

\section{REFERÊNCIAS}

Angelini, A. L., Alves, I. C. B., Custódio, E. M., Duarte, W. F., \& Duarte, J. L. M. (1999). Manual de Matrizes Progressivas Coloridas de Raven - Escala Especial. São Paulo: Centro Editor de Testes de Pesquisas em Psicologia.

Bandura, A. (1977). Self-efficacy: Toward a unifying theory of behavioral change. Psychological Review, 84(2), 191-215.

Batista, C. G. (1999). Observação dos comportamentos sociais de crianças com deficiência visual. Em F. P. N. Sobrinho \& A. C. B. Cunha (Orgs.), Dos problemas disciplinares aos distúrbios de conduta (pp. 113-131). Rio de Janeiro: Qualitymark.

Boruchovitch, E. (1994). As variáveis psicológicas e o processo de aprendizagem: Uma contribuição para a psicologia escolar. Psicologia: Teoria e Pesquisa, 10(1), 129-139.

Boruchovitch, E. (2001). Dificuldades de aprendizagem, problemas motivacionais e estratégias de aprendizagem. Em E. Boruchovitch, F. F. Sisto, L. D. T. Fini, R. P. Brenelli \& S. C. Martinelli (Orgs.), Dificuldades de aprendizagem no contexto psicopedagógico (pp. 40-59). Petrópolis: Vozes.

Boruchovitch, E (2008). A motivação para aprender de estudantes em cursos de formação de professores. Educação, 31(1), 30-38.

Costa, E. R., \& Boruchovitch, E. (2000). Fatores que influenciam no uso de estratégias de aprendizagem, Psico-USF, 5(1), 11-24.

Cruvinel, M., \& Boruchovitch, E. (2004). Sintomas depressivos, estratégias de aprendizagem e rendimento escolar de alunos do ensino fundamental. Psicologia em Estudo, 9(3), 369-378.

Danna, M. F., \& Matos, M. A. (1982). Ensinando observação: Uma introdução. São Paulo: EDICON.

Enumo, S. R. F., \& Batista, C. G. (2006). Jogo de Perguntas de Busca com Figuras Geométricas para crianças com Deficiência Visual (PBFG-DV). Em M. B. M. Linhares, A. C. M. Escolano \& S. R. F. Enumo (Orgs.), Avaliação assistida: Fundamentos, procedimentos e aplicabilidade (pp. 39-43). São Paulo: Casa do Psicólogo.

Fagundes, A. J. F. M. (1982). Descrição, definição e registro de comportamento. São Paulo: EDICON.

Ferrão, E. S., \& Enumo, S. R. F. (2008). To what extent are non-intellectual factors included in current approaches to dynamic assessment: A review. Educational and Child Psychology, 25(1), 59-65.

Feüerstein, R., Rand, Y., \& Hoffman, M. B. (1979). The dynamic assessment of retarded performers: The learning potential assessment, device theory, instrument and techniques. Baltimore: University Park Press.

Feüerstein, R., Rand, Y. Hoffman, M. B., \& Miller, R. (1980). Instrumental enrichment: An intervention program for cognitive enrichment. Baltimore: University Park Press. 
Haywood, H. C. (2008). Twenty years of IACEP, and a focus on dynamic assessment: Progress, problems, and prospects. Journal of Cognitive Education and Psychology, 7(3), 419-442. Retirado em 25 de abril de 2009, de http://www.coged.org/ journal/v7i3/

Haywood, H. C., \& Lidz, C. S. (2007). Dynamic assessment in practice. New York: Cambridge University Press.

Haywood, H. C., \& Tzuriel, D. (2002). Applications and challenges in dynamic assessment. Peabody Journal of Education, 77(2), 40-63.

Jensen, M. R. (1997). Proximal elements of autoplastic enhancement. Atlanta, GA: Cognitive Education Systems.

Lidz, C. S. (1987). Dynamic assessment: An interactional approach to evaluating learning potencial. London: Guilford Press.

Lidz, C. S. (1991). Practitioner's guide to dynamic assessment. New York: The Guilford Press.

Lidz, C. S. (2000). The Application of Cognitive Functions Scale (ACFS): An example of curriculum-based dynamic assessment. Em C. S. Lidz \& J. G. Elliot (Orgs.), Dynamic assessment: Prevailing models and applications (pp. 407-439). Amsterdam: JAI/Elsevier Science.

Lidz, C. S. (2003). Early childhood assessment. New York: Wiley \& Sons.

Linhares, M. B. M., \& Enumo, S. R. F. (2007). Avaliação assistida de crianças no Brasil. Em J. C. Alchieri (Org.), Avaliação psicológica: Perspectivas e contextos (pp. 67-113). São Paulo: Vetor Editora.

Linhares, M. B. M., Escolano, A. C. M., \& Enumo, S. R. F. (Orgs.) (2006). Avaliação assistida: Fundamentos, procedimentos e aplicabilidade. São Paulo: Casa do Psicólogo.

Linhares, M. B. M., \& Gera, A. A. S. (2006). Jogo de Perguntas de Busca com Figuras Diversas (PBFD). Em M. B. M. Linhares, A. C. M. Escolano \& S. R. F. Enumo (Orgs.), Avaliação assistida: Fundamentos, procedimentos e aplicabilidade (pp. 45-48). São Paulo: Casa do Psicólogo.

Linhares, M. B. M., Santa Maria, M. R., \& Escolano, A.C.M. (2006). Indicadores de desempenho cognitivo na resolução dos problemas de pergunta de busca - Tipos de perguntas e solução. Em M. B. M. Linhares, A. C. M. Escolano \& S. R. F. Enumo (Orgs.), Avaliação assistida: Fundamentos, procedimentos e aplicabilidade (pp. 49-52). São Paulo; Casa do Psicólogo.

Loureiro, S. R. (2000). Aprendizagem escolar: Avaliação de aspectos afetivos. Em C. A. R. Funayama (Org.), Problemas de aprendizagem: Enfoque multidisciplinar (pp. 65-76). Campinas: Átomo \& Alínea.

Neves, L. F. (2002). Um estudo sobre as relações entre a percepção e a expectativa dos professores, dos alunos e o desempenho acadêmico em matemática. Dissertação de Mestrado não-publicada, Universidade Estadual de Campinas.

Paula, K. M. P. (2004). Avaliação assistida: Análise de indicadores cognitivos, comportamentais e afetivo-motivacionais em crianças na situação de intervenção com sistema de comunicação alternativa. Tese de Doutorado não-publicada, Universidade Federal do Espírito Santo.

Paula, K. M. P., \& Enumo, S. R. F. (2007). Avaliação assistida e comunicação alternativa: Procedimentos para a educação inclusiva. Revista Brasileira de Educação Especial, 13(1), 3-26.
Pintrich, P. (2003). A motivational science perspective on the role of student motivation in learnig and teaching contexts. Journal of Education Psychology, 95(4), 667-686.

Santa Maria, M. R., \& Linhares, M. B. M. (1999). Avaliação cognitiva assistida de crianças com indicações de dificuldade de aprendizagem escolar e deficiência mental leve. Psicologia: Reflexão e Crítica, 12(2), 395-417.

Santana, P. R., Otta, E., \& Bastos. M. F. (1993). Um estudo naturalístico de comportamentos empáticos em pré-escolares. Psicologia: Teoria e Pesquisa, 9(3), 575-586.

Sigolo, S. R. R. L., \& Alves, Z. M. M. B. (1998). Análises de dados de interação mãe-criança: Construção de sistemas de categorias. Em G. Romanelli \& Z. M. M. Biasoli-Alves (Orgs.), Diálogos metodológicos sobre prática de pesquisa (pp. 87118). Ribeirão Preto: Legis Summa.

Silva, M. P. V., \& Salomão, N. M. R. (2002). Interações verbais e não-verbais entre mães-crianças portadoras de Síndrome de Down e entre mães-crianças com desenvolvimento normal. Estudos de Psicologia, 7(2), 311-323.

Skuy, M., \& Skuy, M. (2005). Contribuition of intelligence and cognitive-affective variables to university grades african, indian, and white engineering students in South Africa. Journal of Cognitive Education and Psychology, 4(3), 25-46. Retirado em 12 de dezembro de 2005 de http://www.coged.org/journal/ V5I1/

Stein, L. M. (1994). TDE - Teste de Desempenho Escolar: Manual para aplicação e interpretação. São Paulo: Casa do Psicólogo.

Sternberg, R., \& Grigorenko, E. (2002). Dynamic testing: The nature and measurement of learning potential. Cambridge: Cambridge University Press.

Trentacosta, C. J., \& Izard, C. E. (2007). Kindergarten children's emotion competence as a predictor of their academic competence in first grade. Emotion, 7(1), 77-88.

Tzuriel, D. (1991). Cognitive modifiability mediated learning experience and affective-motivational processes: A transactional approach. Em R. Feuerstein, P. S. Klein \& A. Tannenbaum (Orgs.), Mediated learning experience: Theoretical, psychosocial and learning implications (pp. 95-120). London: Freund.

Tzuriel, D. (2001). Dynamic assessment of young children. New York: Kluwer Academic/Plenum Plublishers.

Tzuriel, D., \& Samuels, M. (2000). Dynamic assessment of learning potential: Inter-rater reability of deficiency cognitive functions, types of mediation, and non-intellective factors. Journal of Cognitive Education and Psychology, 1(1), 41-64. Retirado em 25 de janeiro de 2004, de http://www.coged.org/ journal/v1i1/

Tzuriel, D., Samuels, M. T., \& Feuerstein, R. (1988). Non-intellective factors in dynamic assessment. In R. M. Gupta \& P. Coxhead (Orgs.), Cultural diversity and learnig efficiency: Recent developments in assessment (pp. 141-163). London: NFER-Nelson.

Zigler, E., Bennett-Gates, D., Hodapp, R., \& Henrich, C. C. (2002). Assessing personality traits of individuals with mental retardation. American Journal of Mental Retardation, 107(3), 181-193.

Weiner, B. (1990). History of motivational research in education. Journal of Educational Psychology, 82(4), 616-622. 
Recebido: $23 / 08 / 2007$

Última revisão: $13 / 06 / 2010$

Aceite final: $20 / 07 / 2010$

Nota:

${ }^{1}$ Este artigo é baseado em parte da tese de doutorado da primeira autora, realizada e apresentada no Curso de Pós-graduação em Psicologia da Universidade Federal do Espírito Santo sob a supervisão da terceira e quarta autoras.

\section{Sobre as autoras:}

Erika da Silva Ferrão: Psicóloga, doutora em Psicologia pela Universidade Federal do Espírito Santo, professora do Centro Universitário Vila Velha (UVV).

Elissa Orlandi Moraes: Psicóloga, mestre em Psicologia pela Universidade Federal do Espírito Santo.

Sônia Regina Fiorim Enumo: Psicóloga, doutora em Psicologia Experimental pela Universidade de São Paulo, professora do Programa de Pós-graduação em Psicologia da Universidade Federal do Espírito Santo. Bolsista de produtividade em pesquisa 1B do CNPq.

Maria Beatriz Martins Linhares: Psicóloga, doutora em Psicologia Experimental pela Universidade de São Paulo, professora da Faculdade de Medicina de Ribeirão Preto da Universidade de São Paulo. Bolsista de produtividade em pesquisa 1A do CNPq.

Gizele Pereira de Sousa: Psicóloga, ex-bolsista de IC (CNPq).

Endereço para correspondência: Erika da Silva Ferrão - Rua Ceará, 155 - apto. 306 - Bairro Praia da Costa - 29101-291 Vila Velha/ES. Endereço eletrônico: edsferrao@yahoo.com.br. 